\title{
Matching in Frequent Tree Discovery
}

\author{
Björn Bringmann \\ Machine Learning Lab, University of Freiburg \\ Georges-Köhler-Alle, Geb. 079, 79098 Freiburg, Germany \\ bbringma@informatik.uni-freiburg.de
}

\begin{abstract}
Various definitions and frameworks for discovering frequent trees in forests have been developed recently. At the heart of these frameworks lies the notion of matching, which determines when a pattern tree matches a tree in a data set. We introduce a novel notion of tree matching for use in frequent tree mining and we show that it generalizes the framework of Zaki while still being more specific than that of Termier et al. Furthermore, we show how Zaki's TreeMinerV algorithm can be adapted towards our notion of tree matching. Experiments show the promise of the approach.
\end{abstract}

\section{Introduction}

In the past few years, interest has grown in extending the frequent item set paradigm to more expressive pattern types such as graphs, trees and sequences. Special attention has been devoted to semi-structured and tree-structured data $[3,5,7,2,6,1,8]$. Most of these approaches aim at finding all frequent trees in a forest of trees. They differ in the algorithms and implementation details, and more importantly also in the underlying notion of tree matching. When does one tree match another one? Asai et al., Zaki and Termier et al. $[1,8,6]$ provide different answers to this question. Asai's notion is more restrictive than Zaki's, which is in turn more restrictive than Termier's. Termier et al. have also shown that it can be beneficial to work with more permissive notions of matching. However, this typically comes at a computational price. Indeed, due to the expressiveness of their framework, Termier et al. cannot guarantee completeness, whereas Zaki and Asai et al. do.

The key contribution of this paper is a novel notion of tree matching. It fills the gap between the approaches of Zaki and Termier et al. . We also argue that - in the context of web mining - it is a natural way to look at tree matching. Furthermore, we extend Zaki's algorithm, introduce a new pruning technique, and experimentally evaluate our approach.

\section{Frequent Tree Mining}

A rooted $k$-tree $t$ is a set of $k$ nodes $V_{t}$ where each $v \in V_{t}$, except one called root, has a parent denoted $\pi(v) \in V_{t}$. We use $\lambda(v)$ to denote the label of a node and an operator $\prec$ to denote the order from left to right among the children of a node. The transitive closure of $\pi$ will be denoted $\pi^{*}$. Let $\mathcal{L}$ be a formal language composed of all labeled, ordered, rooted trees and $\mathcal{D} \subset \mathcal{L}$ a database. To count trees $t \in \mathcal{D}$ containing a pattern $p$ we define a function $d_{t}: \mathcal{L} \rightarrow\{0,1\}$ to be 1 iff $p$ matches the tree $t$ and 0 otherwise. The frequency of $p$ in $\mathcal{D}$ can then be defined as $\sigma_{\mathcal{D}}(p)=_{\text {def }} \Sigma_{t \in \mathcal{D}} d_{t}(p)$. Given a set of trees $\mathcal{D}$ and a minimum frequency $\alpha$, the task of tree-mining is to find all patterns $p$ such that $\sigma_{\mathcal{D}}(p) \geq \alpha$.

Tree Matching. Termier et al. [6] present three notions of tree matching. Formally speaking:

Definition $1 A$ tree $t$ matches a tree $t^{\prime}$ iff a mapping $\varphi: V_{t} \rightarrow V_{t^{\prime}}$ exists such that

$\forall u, v \in V_{t}: \lambda(u)=\lambda(\varphi(u)) \wedge u \prec v \Leftrightarrow \varphi(u) \prec \varphi(v)$.

Now, each of the notions of matching imposes further constraints for two trees to match. More specifically, tree inclusion requires $\forall u, v \in V_{t}: \pi(u)=v \Leftrightarrow \pi(\varphi(u))=\varphi(v)$. The more relaxed notion of tree embedding requires $\forall u, v \in V_{t}: \pi^{*}(u)=v \Leftrightarrow \pi^{*}(\varphi(u))=\varphi(v)$. Finally, in tree subsumption there has to be a mapping $\varphi: V_{t} \rightarrow 2^{V_{t^{\prime}}}$ from $V_{t}$ to the powerset of $V_{t^{\prime}}$ preserving the labels and $\forall u, v \in V_{t}: \pi^{*}(u)=v \Rightarrow \pi^{*}(\varphi(u))=\varphi(v)$. This notion does not preserve the order of the child nodes.

The novel notion of tree matching, to which we will refer as tree incorporation, is more relaxed than tree embedding since a $\pi^{*}$ relationship in the data does not have to hold in the pattern. It is more restrictive than tree subsumption as $\varphi$ is a mapping to $V_{t^{\prime}}$ rather than to $2^{V_{t^{\prime}}}$, and it attempts to preserve the order of the children. More formally speaking:

Definition 2 A tree $t$ is incorporated in a tree $t^{\prime}$ iff there exists a mapping $\varphi: V_{t} \rightarrow V_{t^{\prime}}$ such that

$$
\begin{aligned}
\forall u, v \in V_{t} & : \quad \lambda(u)=\lambda(\varphi(u)) \\
\wedge & u \prec v \Leftarrow \varphi(u) \prec \varphi(v) \\
& \wedge \pi^{*}(u)=v \Rightarrow \pi^{*}(\varphi(u))=\varphi(v) .
\end{aligned}
$$


The relation among the different notions of matchings is captured by the following theorem (proof omitted).

Theorem 1 For all trees $t, t^{\prime}$, $t$ is included in $t^{\prime} \Rightarrow t$ is embedded in $t^{\prime} \Rightarrow$ $t$ is incorporated in $t^{\prime} \Rightarrow t$ is subsumed by $t^{\prime}$.

As a consequence, the set of frequent trees w.r.t. a data set $\mathcal{D}$ for tree inclusion is smaller than for tree embedding, which is in turn smaller than for tree incorporation and this one is again smaller w.r.t. tree subsumption. This motivates the use of the notion of matching as a parameter of frequent tree discovery tasks. Basically our novel definition fills the gap between tree embedding and tree subsumption. The toy-example of an online shop (figure 1) compares tree

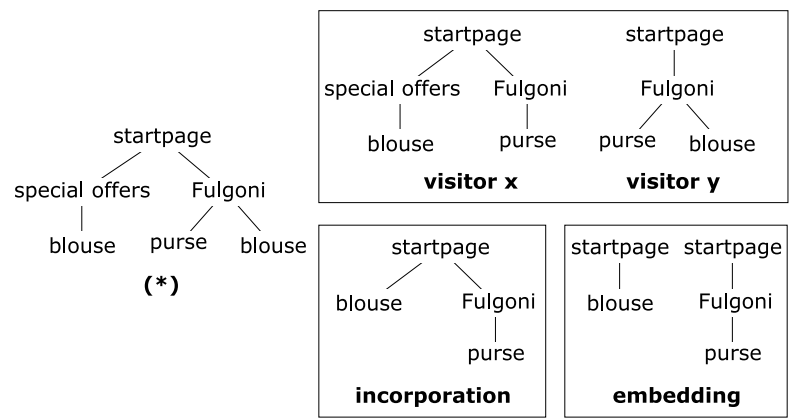

Figure 1. Two subtrees from a master-tree $\left(^{*}\right)$ and respective most-specific patterns

incorporation and embedding. While the novel definition yields only one most specific pattern, the notion of tree embedding yields two. According to tree embedding some visitors looked at the blouse and some at the Fulgoni purse. The single most specific pattern according to the novel notion offers more information: The visitors looking at the blouse and the visitors looking at the purse are the same persons. This knowledge might be helpful when restructuring the online-shop to improve accessibility or placement of advertisements. Technically, incorporated trees can be discovered by an algorithm similar to the fast and complete TreeMinerV algorithm [8]. In our implementation the user can select between the notions of tree inclusion, embedding, and incorporation as a constraint similar to minimum frequency. A further optimization of the algorithm is described in section 3 .

\section{Frequent Tree Mining Algorithms}

In this section, we discuss frequent tree mining w.r.t. tree incorporation. We explain candidate tree generation, candidate counting, and a novel pruning technique.

Candidate Tree Generation. To generate candidate patterns we use a method called rightmost expansion to canonically enumerate all labeled, ordered, rooted trees. This technique was independently proposed by Zaki and Asai et al.

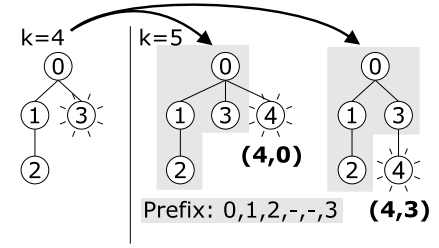

Figure 2. Rightmost expansion and equivalence classes $(\mathrm{EQ})$.

$[8,1]$. It works in a levelwise manner adding a single node to a known frequent pattern in such a way that every possible candidate pattern will be generated exactly once. Basically a $k$-tree is expanded to several $k+1$-trees by adding new nodes only to its rightmost path as shown in figure 2 . The new node $n_{k+1}$ is called rightmost leaf (RML) and the subtree without its RML is called prefix of the tree. For efficient candidate generation the anti-monotonicity of frequent patterns is used (i.e. a specialization $s$ of a pattern $p$ is not more frequent than $p$ ). Thus, we consider only frequent patterns for extension. With focus on the rightmost extension, patterns are organized in so called equivalence classes $(\mathrm{E} Q)$. An EQ contains all patterns that have the same prefix, i.e. differ only in their RMLs. It contains the pattern prefix only once and for each pattern $P_{i}$ a tuple $\left(\lambda\left(\mathrm{RML}_{i}\right), \pi\left(\mathrm{RML}_{i}\right)\right)$. At this point it is important to note that in the same $E Q$ every pattern $(\lambda, j)$ with $j>p$ is a specialization of a pattern $(\lambda, p)$ with respect to the novel definition. Hence, a $\varphi$ exists that maps the nodes of $(\lambda, p)$ to the nodes of $(\lambda, j)$ with respect to definition 2 . For example the left 5 -tree in figure 2 is a pattern for the right 5-tree, but not vice versa. Due to this relationship there is a large number of generalizations for every pattern. By using the notion of extended instance lists which we will define below, the algorithm generates only the necessary ones to find all patterns of the most specific border (s-set). The resulting pattern set is a superset of the s-set w.r.t. the notion of tree incorporation.

To efficiently count the support of a pattern the algorithm needs information about the instances of the pattern in the data. Let pattern $X$ be a $k$-subtree occurring in a tree $T, \varphi$ the mapping from the pattern-nodes to the nodes of $T$, and $x_{k}$ refers to the RML of $X$. Following Zaki, we use $\mathcal{I}(X)$ to refer to the instance-list (i.e. scope-list) of $X$. Each element of $\mathcal{I}$ is a triple $(t, s, m)$ identifying an instance of $X . t$ is the id of a tree $T$ in which $X$ occurs, $m={ }_{\text {def }}\left\{\varphi(n) \mid n \in\left(X \backslash x_{k}\right)\right\}$ is called match label of the prefix of $X$, and $s$ is the scope of the rightmost leaf $\varphi\left(x_{k}\right)$. These instance lists contain all instances of a pattern w.r.t. tree embedding. For the novel definition, we introduce the notation of extended instance lists $\mathcal{I}^{*}(X)=\mathcal{I}^{*}(\lambda, p)={ }_{\text {def }} \cup_{j \geq p} \mathcal{I}(\lambda, j)$ containing all instances that support a pattern $X$. When two patterns $A$ and $B$ of the same $\mathrm{EQ}$ are joined, the information in the instance lists $\mathcal{I}^{*}(A), \mathcal{I}^{*}(B)$ is used to track the patterns in the same way as described in [8]. Only in the case of an outscope- 


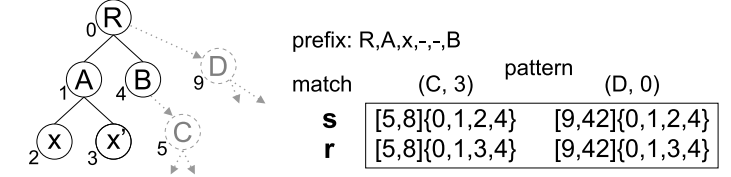

Figure 3. Instances $s$ or $r$ would be removed by instance pruning.

join the condition has to be changed, such that a join is also possible if a new node $D$ is a descendant or left-sibling of $A$, not only if $D$ is a left-sibling of $A$. The interested reader is referred to [8] for more information.

Instance Pruning. Besides simpler pruning techniques like node pruning and edge pruning that are also used by Zaki [8] and Termier et al. [6] we introduce a new technique called instance pruning (IP) that reduces the average computation time to $50 \%$. It is not only applicable to the algorithm working on the novel pattern definition, but also to the algorithm provided by Zaki. As stated before, $d_{t}(X)$ returns a 1 if there is at least one occurrence of pattern $X$ in tree $t$, otherwise it returns 0 . Hence, the frequency of a pattern depends only in part on the number of instances. If there are different instances of a pattern $(x, i)$ in tree $t$, they are represented in the instance list $\mathcal{I}(x, i)$ as $I_{1,0}=\left(t, a_{0}, s\right)$ and $I_{2,0}=\left(t, b_{0}, r\right)$. If the pattern $(x, i)$ is joined with another pattern $(y, j)$ all instances in $I_{1}$ and $I_{2}$ will be joined with the respective instances of $(y, j)$. Consider two groups of instances of the tree $t$ with match labels $r, s$ and triples $\left(t, a_{0 \cdots m}, s\right),\left(t, b_{0 \cdots n}, r\right)$, as shown in figure 3. If for every triple $\left(t, b_{k}, r\right)$ there exists a triple $\left(t, a_{l}, s\right)$ with $a_{l}=b_{k}$, all triples with the match label $r$ can be removed from the $\mathrm{EQ}$. This is possible, as for instances in the same tree with the same match label, only the nodes $a_{l}$ (or $b_{k}$ ) are of relevance for the extension of the instances. If a match label $s$ includes all nodes $b_{k}$ of a match label $r$, no instance can be created out of instances with match label $r$ that cannot be created out of instances with match label $s$. This reduction of instances can efficiently reduce the memory footprint of the process. Even more important than the substantially lowered memory usage is the reduction in computation time. Not only the removed instances themselves are not joined anymore, but also the ones that would have been created by joining them. When the number of labels is low in relation to the number of nodes in the trees, $I P$ can reduce the computation time even to $20 \%$.

Algorithm. Figure 4 shows the algorithm used to compute frequent patterns w.r.t. tree incorporation. As stated before, the algorithm is an extended version of Zaki's TreeMinerV algorithm. The main differences are the usage of the extended instance lists and a new condition for the out-scope test. The sets containing all frequent 1-trees (i.e. nodes) and 2-trees are computed before the main loop. The function Enumerate-Frequent-Subtrees generates all possible refinements of patterns in an $\mathrm{EQ}[P]$. This is done by joining
MineFrequentTrees (D , minsup $)$ :

$F_{1}=\{$ frequent 1 -subtrees $\}$;

$F_{2}=\left\{\right.$ classes $[P]_{1}$ of frequent 2 -subtrees $\}$;

for all $[P]_{1} \in F_{2}$ do Enumerate-Frequent-Subtrees $\left([P]_{1}\right)$;

ENUMERATE-FREQUENT-SUBTREES $([P])$ :

for each element $(x, i) \in[P]$ do $\left[P_{x}\right]=\emptyset$

for each element $(y, j) \in[P]$ with $i \geq j$ do

$R=\{(x, i) \otimes(y, j)\}$

$\mathcal{I}(R)=\left\{\mathcal{I}^{*}(x, i) \cap_{\otimes} \mathcal{I}^{*}(y, j)\right\}$;

if for any $p \in R, p$ is frequent then $\left[P_{x}\right]=\left[P_{x}\right] \cup\{p\}$;

Enumerate-Frequent-Subtrees $\left(\left[P_{x}\right]\right)$;

\section{Figure 4. TreeMining Algorithm}

every pair $(x, i) \otimes(y, j)$ of patterns in $[P]$ including selfjoins. Due to the rightmost expansion it is not allowed to join $(x, i) \otimes(y, j)$ with $i<j$ which would result in noncanonical expansions. A join results in one or two new patterns $(R)$. Afterwards the according instance lists are created by joining the instance lists of the patterns $(x, i)$ and $(y, j)$. Any new pattern that turns out to be frequent is then added to the new $\mathrm{EQ}\left[P_{x}\right]$. If all frequent patterns of the new $\mathrm{EQ}\left[P_{x}\right]$ are computed further refinements of these patterns are generated. Thus, the algorithm proceeds depth-first.

\section{Experimental Results}

A number of experiments were conducted on real-world and synthetic datasets. The real-world dataset (legcare [4]) consists of an online shop's weblog, containing 234942 visits. Each visit is regarded as a subtree of the hierarchically structured website. There where 694 unique labels for the database. For the synthetic dataset we implemented a data generator as described by Zaki [8]. All the experiments were performed on a $3.2 \mathrm{GHz}$ Intel Pentium 4 with $2 \mathrm{~GB}$ main memory, running SUSE 9.0. The algorithms were implemented in $\mathrm{C}++$. For the tree embedding and tree incorporation, instance pruning is available. We compared the number of frequent patterns found by the algorithms and the size of the s-set on both datasets with different minimum support. To calculate the s-set an additional postprocessing step was performed. Figure 5 shows the number of patterns generated during the search and the number of patterns contributing to the s-set for tree embedding and tree incorporation on the legcare dataset. As mentioned earlier, our novel definition is more relaxed than tree embedding such that more patterns are generated and discovered during the search. In contrast to the figures shown here (figure 5), there is no order between the notions w.r.t. the size of the according s-set. All three notions have in common that they grow exponentially in the number of most specific patterns as well as in patterns considered during the search when reducing the minium support. For the legcare-dataset there was no effect on computation time with and without $I P$. However, using $I P$, the memory consumption dropped 

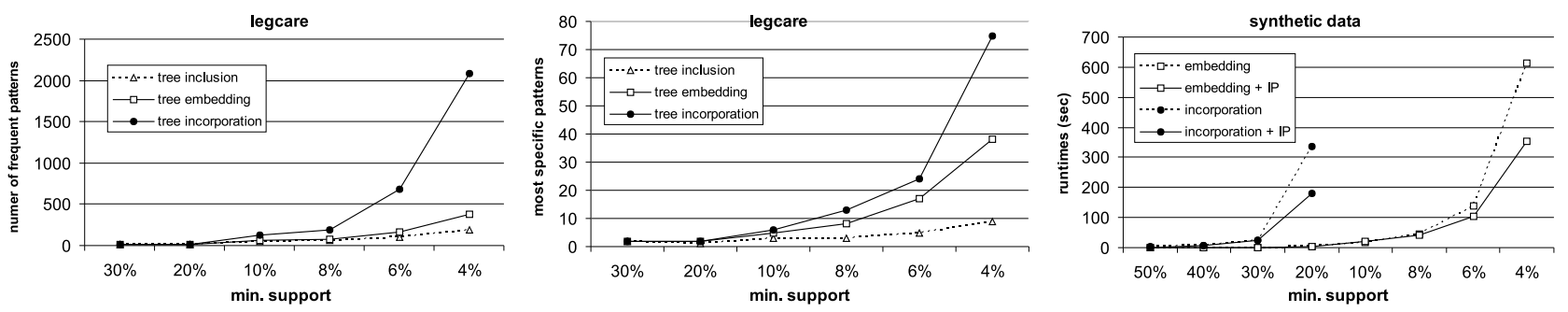

Figure 5. Comparing embedding and incorporation w.r.t. patterns found, s-set size, and $I P$.

dramatically for computing frequent pattern sets with minimum support below $10 \%$. For the experiments with the synthetic data, a master-tree with 100 unique labels and 10000 nodes was generated with a maximum depth and fanout of 10. From this hypothetical website we generated 10000 visits, each a smaller subtree of the master-tree, as database. The right graph in figure 5 shows the results of experiments on this dataset regarding computation time and the effect of instance pruning. The plot clearly indicates an exponential growth in computation time when lowering the minimum support. The solid lines depict the required time with $I P$. Both, tree embedding and tree incorporation, show a significant speedup for low minimum support levels with $I P$.

\section{Conclusions and Related Work}

The algorithm presented in this paper improves and expands the TreeMinerV algorithm with our novel pattern definition. The introduced instance pruning reduces the computation time as well as the memory usage of the algorithm in many cases. Unfortunately we are not yet able to deal with low minimum support levels. Since the amount of frequent patterns in large databases grows fast when lowering the minimum support, it appears to be useful to calculate only the set of all most specific patterns. Until now an additional post processing step is necessary to do this which requires additional computation time. We hope to be able to incorporate this step directly into the mining process to reduce the post processing time. Furthermore, it would be nice to have more constraints, such as a most-general or most-specific pattern, to enable the user to focus the search as in [2] or to extend the tree-mining process to first order logic. On the other hand the frequent patterns discovered could also be used as features for some classifier as in [9]. Considering our novel notion we still have to evaluate if the additional cost in time and memory is justified by 'better' patterns. Finally, it depends on the data and on the requirements of the user which tree matching notion is the best.

Related Work. The most directly related work to this paper is Zaki's TreeMinerV as well as Termier's TreeFinder and FreqT by Asai $[8,6,1]$. Zaki uses a smart, so called vertical representation to facilitate the candidate count enabling a fast mining process that scales well, even with large datasets. We adopted this idea for our pattern definition, which is more specific than tree subsumption used by Termier. Since our definition is more general than Zaki's we find much more patterns than with tree embedding. Therefore, it is not surprising that the algorithm is slower than TreeMinerV. Using our algorithm to mine embedded trees there is a significant speedup compared to TreeMinerV due to our pruning technique. Compared to TreeFinder, it uses a less general pattern definition, but is complete.

Other algorithms like AGM [3], FSM [5] and gSpan [7] work on graphs rather than trees. They are restricted to subgraphs consisting of edges and if applied to trees would only discover frequent trees in the sense of subtree inclusion.

Acknowledgments. The real world dataset was kindly provided by Blue Martini Software. The author thanks Luc De Raedt for encouragement for writing this paper and Mohammed Zaki for providing the TreeMiner source code. Also many thanks to Andreas Karwath, Albrecht Zimmermann, and the anonymous reviewers whose comments helped to improve the paper. This research was supported by the European Project: IST-2000-26469, cInQ.

\section{References}

[1] T. Asai, K. Abe, S. Kawasoe, H. Arimura, H. Sakamoto, and S. Arikawa. Effi cient substructure discovery from large semistructured data. In Proc. of SIAM SDM, pages 158-174, 2002.

[2] L. De Raedt and S. Kramer. The levelwise version space algorithm and its application to molecular fragement fi nding. In Proc. of IJCAI-01, pages 853-862, 2001.

[3] A. Inokuchi, T. Washio, and H. Motoda. An apriori-based algorithm for mining frequent substructures from graph data. In Proc. of PKDD, pages 13-23, 2000.

[4] R. Kohavi, C. Brodley, B. Frasca, L. Mason, and Z. Zheng. KDD-Cup 2000 organizers' report: Peeling the onion. SIGKDD Explorations, 2(2):85-98, 2000.

[5] M. Kuramochi and G. Karypis. Frequent subgraph discovery. In Proc. of ICDM, 2001.

[6] A. Termier, M.-C. Rousset, and M. Sebag. Treefi nder: a fi rst step towards xml data mining. In Proc. of ICDM, 2002.

[7] X. Yan and J. Han. gspan: Graph-based substructure pattern mining. In Proc. of ICDM, 2002.

[8] M. Zaki. Effi ciently mining frequent trees in a forest. In Proc. of KDD, 2002.

[9] M. J. Zaki and C. C. Aggarwal. Xrules: An effective structural classifi er for xml data. In Proc. of SIGKDD 03, 2003. 